\title{
Entropy generation in a pipe due to non-Newtonian fluid flow: Constant viscosity case
}

\author{
M PAKDEMIRLI $^{1}$ and B S YILBAS ${ }^{2, *}$ \\ ${ }^{1}$ Department of Mechanical Engineering, Celal Bayar University, Manisa, Turkey \\ ${ }^{2}$ Department of Mechanical Engineering, King Fahd University of Petroleum and \\ Minerals, P.O. Box 1913, Dhahran 31261, Saudi Arabia \\ e-mail: mpak@kfupm.edu.sa; bsyilbas@kfupm.edu.sa
}

MS received 20 March 2005; revised 30 August 2005

\begin{abstract}
Non-Newtonian fluid flow in a pipe system is considered and a third grade non-Newtonian fluid is employed in the analysis. The velocity and temperature distributions across the pipe are presented. Entropy generation number due to heat transfer and fluid friction is formulated. The influences of non-Newtonian parameter and Brinkman number on entropy generation number are examined. It is found that increasing the non-Newtonian parameter reduces the fluid friction in the region close to the pipe wall. This in turn results in low entropy generation with increasing non-Newtonian parameter. Increasing Brinkman number enhances the fluid friction and heat transfer rates; in which case, entropy number increases with increasing Brinkman number.
\end{abstract}

Keywords. Non-Newtonian; fluid flow; pipe; entropy; entropy number.

\section{Introduction}

Non-Newtonian fluid flowing through pipes is of importance for many engineering applications, some of which include chemical engineering operations, polymer processing, petrochemical applications etc. Moreover, two-phase flows, consisting of non-condensing substrates such as fluid and solid particles, are involved with complex physical situations. In this case, the resulting flow structure depends on fluid properties as well as size, distribution and amount of solid particles in the flow system. To tackle such situations, separate flow equations for each phase can be imposed, provided that the governing equations are coupled through conservation equations. This requires excessive computational effort and analytical solutions for the coupled equations are almost impossible to obtain. However, a rather simple approach can be introduced to consider the mixture as a single homogeneous continuum. In this case, the mixture of fluid and solid particles behaves like a non-Newtonian fluid (Johnson et al 1991).

Considerable research studies were carried out to investigate non-Newtonian fluid flows in pipes. Ekmann et al (1986) studied laminar flow of highly loaded suspensions in horizontal

\footnotetext{
*For correspondence
} 
pipes. They indicated that although the power-law model adequately agreed with the shear stress and shear rate measurements for non-Newtonian fluids, it could not predict the pressure loss in the pipe during the transport of a coal-liquid mixture in a fuel delivery system. In this case, it was shown that the power-law model was not capable of predicting the normal stress effects that lead to phenomena like die-swell and rod-climbing (Dunn \& Fosdick 1974). Moreover, employment of second and third grade fluid models becomes appropriate to predict the flow structures for such flow situations (Fosdick \& Rajagopal 1980). Massoudi and Christie (1995) investigated the effects of variable viscosity and viscous dissipation on the flow of a third grade fluid in a pipe. They found that the viscosity has significant influence on the temperature and velocity distribution in the pipe. The approximate analytical solution for temperature and velocity profiles due to third-grade fluid flow in pipe was presented by Yurusoy \& Pakdemirli (2002). They used a perturbation method in the analysis. The conventional and multiple deck boundary layer approach for second and third grade fluids were introduced by Pakdemirli (1994). He showed the existence of triple decks for the boundary layer analysis of such fluids.

Thermodynamic irreversibility associated with the flow system gives insight into the frictional and heat transfer losses in the system. Moreover, the entropy generation is associated with the thermodynamic irreversibilities taking place in the system. Consequently, thermodynamic irreversibility can be quantified through entropy calculations. Bejan (1979) presented the mechanisms involving with the entropy generation in applied thermal engineering. Bejan (1994) introduced the concept of entropy generation and minimization in thermal systems. Considerable research studies were carried out to examine entropy generation in flow systems. Yilbas et al (1999) investigated entropy generation in semi-blocked pipes. They showed that the Merit number was dependent on fluid properties. Irreversibility analysis for channel and pipe flows for non-Newtonian fluids was carried out by Mahmud \& Fraser (2002). They presented entropy generation number for both flow situations. The modelling of non-isothermal viscoelastic flows was carried out by Peters \& Baaijens (1997). They discussed the partioning between dissipated and elastically stored energy and the difference between entropy and energy elasticity.

In the present study, a pipe flow of third grade non-Newtonian fluid with external heating is considered. Temperature and velocity distribution in the pipe are presented analytically. Entropy generation in the pipe is formulated and the influence of Brinkman number and nonNewtonian parameter on the entropy generation number is investigated.

\section{Velocity and temperature profiles}

The non-dimensional form of the equations of motion of a third grade fluid in a pipe with heat transfer was derived by Massoudi \& Christie (1995):

$$
\begin{array}{r}
\frac{\mathrm{d} \mu}{\mathrm{d} r} \frac{\mathrm{d} v}{\mathrm{~d} r}+\frac{\mu}{r}\left(\frac{\mathrm{d} v}{\mathrm{~d} r}+r \frac{\mathrm{d}^{2} v}{\mathrm{~d} r^{2}}\right)+\frac{\Lambda}{r}\left(\frac{\mathrm{d} v}{\mathrm{~d} r}\right)^{2}\left(\frac{\mathrm{d} v}{\mathrm{~d} r}+3 r \frac{\mathrm{d}^{2} v}{\mathrm{~d} r^{2}}\right) \\
\frac{\mathrm{d}^{2} \theta}{\mathrm{d} r^{2}}+\frac{1}{r} \frac{\mathrm{d} \theta}{\mathrm{d} r}+\Gamma\left(\frac{\mathrm{d} v}{\mathrm{~d} r}\right)^{2}\left[\mu+\Lambda\left(\frac{\mathrm{d} v}{\mathrm{~d} r}\right)^{2}\right]=0, \\
v(1)=\theta(1)=0, \quad \frac{\mathrm{d} v}{\mathrm{~d} r}(0)=\frac{\mathrm{d} \theta}{\mathrm{d} r}(0)=0,
\end{array}
$$


where $r$ is the dimensionless radius $(0<r<1), v$ is the dimensionless velocity, $\theta$ is the dimensionless temperature and $\mu$ is the dimensionless viscosity. The terms are related to the dimensional ones through the following relations,

$$
r=\frac{\bar{r}}{R}, \quad v=\frac{\bar{v}}{V_{0}}, \quad \theta=\frac{\bar{\theta}-\theta_{w}}{\theta_{m}-\theta_{w}}, \quad \mu=\frac{\bar{\mu}}{\mu_{*}},
$$

where $R$ is the radius, $V_{0}$ is a reference velocity, $\mu_{*}$ is a reference viscosity, $\theta_{m}$ and $\theta_{w}$ are the bulk mean fluid temperature and wall temperature respectively.

The dimensionless parameters involved in (1) and (2) are

$$
C=\frac{C_{1} R^{2}}{\mu_{*} V_{0}}, \quad C_{1}=\frac{\partial p}{\partial z}, \quad \Gamma=\frac{\mu_{*} V_{0}^{2}}{k\left(\theta_{m}-\theta_{w}\right)}, \quad \Lambda=\frac{2 \beta_{3} V_{0}^{2}}{\mu_{*} R^{2}},
$$

where $C_{1}$ is the pressure drop in the axial direction, $\Gamma$ is the Brinkman number, $\Lambda$ is the dimensionless non-Newtonian viscosity, $\beta_{3}$ is the dimensional material constant for the third grade fluid and $k$ is the thermal conductivity.

Approximate solutions for velocity and temperature profiles using perturbation methods have been presented for the above equations by Yurusoy \& Pakdemirli (2002),

$$
\begin{aligned}
& v=-\frac{C}{4}\left(1-r^{2}\right)+\frac{\Lambda C^{3}}{32}\left(1-r^{4}\right), \\
& \theta=\frac{\Gamma C^{2}}{64}\left(1-r^{4}\right)-\frac{\Lambda \Gamma C^{4}}{576}\left(1-r^{6}\right) .
\end{aligned}
$$

The criterion for these solutions to be valid is

$$
\frac{C^{2} \Lambda}{8} \ll 1
$$

\section{Viscous dissipation and entropy generation}

The dimensional viscous dissipation term $(\bar{\phi})$ can be obtained from equations of motion, i.e.:

$$
\bar{\phi}=\bar{\mu}\left(\frac{\mathrm{d} \bar{v}}{\mathrm{~d} \bar{r}}\right)^{2}+2 \beta_{3}\left(\frac{\mathrm{d} \bar{v}}{\mathrm{~d} \bar{r}}\right)^{4}
$$

or inserting the dimensionless quantities yields

$$
\bar{\phi}=\frac{\mu_{*} V_{0}^{2}}{R^{2}}\left(\frac{\mathrm{d} v}{\mathrm{~d} r}\right)^{2}\left[\mu+\Lambda\left(\frac{\mathrm{d} v}{\mathrm{~d} r}\right)^{2}\right] .
$$

The dimensional volumetric entropy generation is defined as (Bejan 1995):

$$
S_{\text {gen }}^{\prime \prime \prime}=\frac{k}{\bar{\theta}_{0}^{2}}\left(\frac{\mathrm{d} \bar{\theta}}{\mathrm{d} \bar{r}}\right)^{2}+\frac{\bar{\phi}}{\bar{\theta}_{0}}
$$


where $\bar{\theta}_{0}$ is the reference temperature. The first term in (11) is the volumetric entropy generation due to heat transfer and the second term is the entropy generation due to viscous dissipation. Substituting (10) into (11), expressing the terms in dimensionless forms, one finally obtains:

$$
N s=\left(\frac{\mathrm{d} \theta}{\mathrm{d} r}\right)^{2}+\theta_{0} \Gamma\left(\frac{\mathrm{d} v}{\mathrm{~d} r}\right)^{2}\left[\mu+\Lambda\left(\frac{\mathrm{d} v}{\mathrm{~d} r}\right)^{2}\right]
$$

where $N s$ is the entropy generation number. It is defined by dividing the dimensional volumetric entropy generation by a reference volumetric entropy generation $S_{G}^{\prime \prime \prime}$. The relevant definitions are:

$$
N s=\frac{S_{\text {gen }}^{\prime \prime \prime}}{S_{G}^{\prime \prime \prime}}, \quad S_{G}^{\prime \prime \prime}=\frac{k\left(\theta_{m}-\theta_{w}\right)^{2}}{\bar{\theta}_{0}^{2} R^{2}} \quad \theta_{0}=\frac{\bar{\theta}_{0}}{\theta_{m}-\theta_{w}} .
$$

In (12), the first term due to heat generation can be assigned as $N s_{1}$ and the second term due to viscous dissipation as $N s_{2}$, i.e.:

$$
N s_{1}=\left(\frac{\mathrm{d} \theta}{\mathrm{d} r}\right)^{2}, \quad N s_{2}=\theta_{0} \Gamma\left(\frac{\mathrm{d} v}{\mathrm{~d} r}\right)^{2}\left[\mu+\Lambda\left(\frac{\mathrm{d} v}{\mathrm{~d} r}\right)^{2}\right] .
$$

Assuming constant viscosity, the non-dimensional viscosity can be taken as one $(\mu=1)$. Since the temperature and velocity profiles are known functions, they can be inserted from (6) and (7) into (12) and (14) for final evaluations of the entropy generation numbers.

\section{Results and discussions}

Non-Newtonian fluid of third grade type flowing in a pipe is considered. Entropy generation in the flow field due to fluid friction and heat transfer is formulated. The influence of Brinkman number and non-Newtonian parameter on the entropy generation number is examined. In the analysis the constant viscosity case is accommodated.

Figure 1 shows the velocity profile and figure 2 shows temperature profiles across the pipe for different non-Newtonian parameters. The non-Newtonian parameter $(\Lambda)$ of zero indicates that the fluid is Newtonian type. Consequently, as non-Newtonian parameter increases, the magnitude of maximum velocity and rate of fluid strain reduce. This indicates that the viscous dissipation in the flow field is higher for a Newtonian fluid than for its counterpart corresponding to non-Newtonian. However, the magnitude of maximum temperature and temperature gradient close to the pipe wall attain lower values for non-Newtonian fluids than for a Newtonian fluid. In this case, for Newtonian fluid, due to the high temperature gradient close to the wall, the heat transfer rate enhances. This, in turn, results in enhancement of the maximum temperature rise in the fluid. Moreover, the flat top of temperature profile widens across the pipe as non-Newtonian parameter increases. This behaviour shows that heat diffusion and convection across the pipe enhance equating temperature across the pipe as non-Newtonian parameter increases.

Figure 3 shows the entropy generation number due to heat transfer across the pipe as the non-Newtonian parameter is variable. Entropy number attains large values in the vicinity of the pipe wall and as the radial distance increases towards the pipe centre, it reduces sharply. 


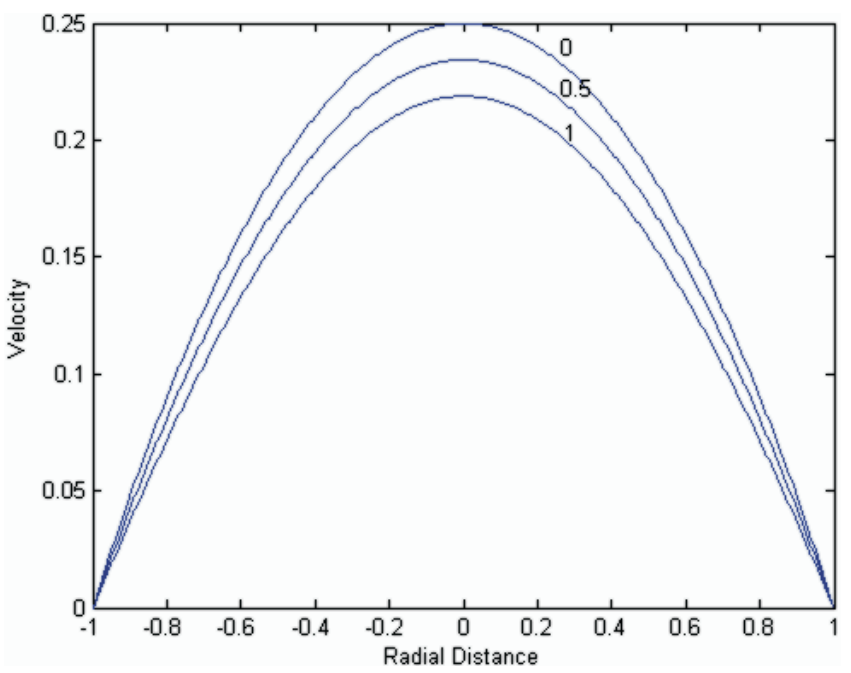

Figure 1. Velocity profiles along the pipe diameter for different non-Newtonian parameter $(\Lambda) \cdot C=-1$.

This is because of the temperature distribution, which becomes almost uniform in the region close to the pipe centre, i.e. temperature gradient reduces to almost zero in this region; consequently, entropy generation reduces to minimum. Increasing non-Newtonian parameter lowers the entropy number close to the pipe wall. In this case, increasing non-Newtonian parameter enhances the diffusional energy transport towards the pipe centre resulting in reduced temperature and temperature gradient in the region close to the pipe wall.

Figure 4 shows entropy generation number due to fluid friction along the pipe cross section. Similar to the case due to heat transfer, entropy generation reduces sharply as the distance increases towards the pipe centre. This is because of sharp decay of velocity gradient in the region close to the pipe wall. At the centre of the pipe, where velocity reaches maximum and its gradient is zero, entropy generation due to fluid friction reduces to zero. However,

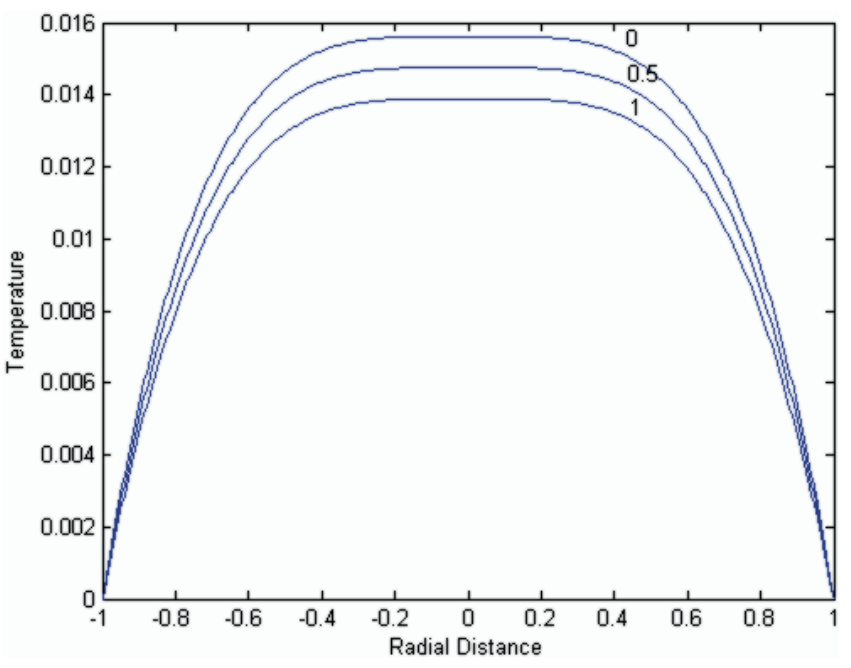

Figure 2. Temperature profiles along the pipe diameter for different non-Newtonian parameters. $\Gamma=1$ and $C=-1$. 


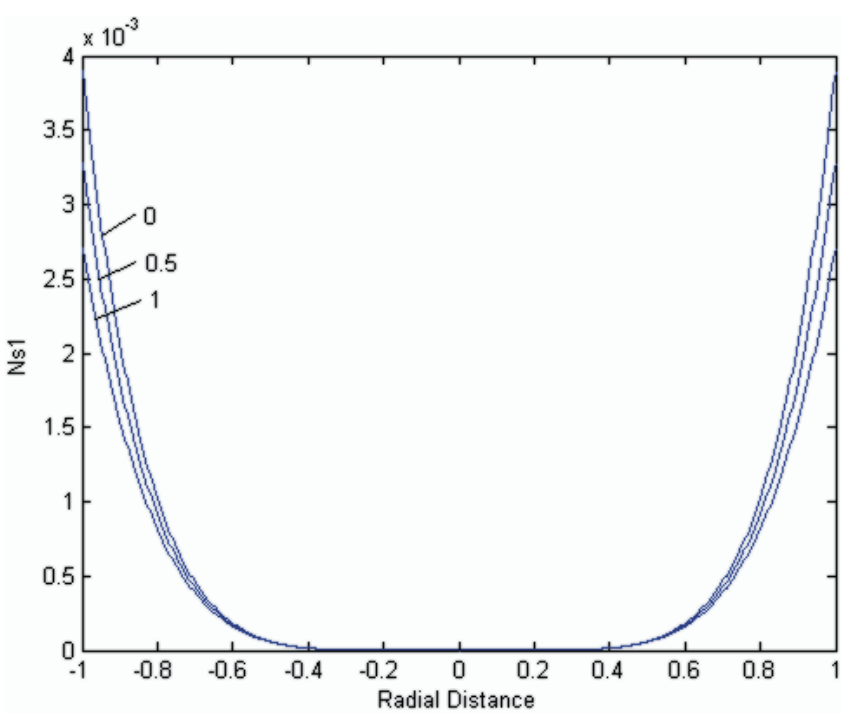

Figure 3. Entropy generation number due to heat transfer along the pipe diameter for different non-Newtonian parameters. $\Gamma=$ 1 and $C=-1$.

increasing non-Newtonian parameter lowers the entropy generation number, which is more pronounced in the region close to the pipe wall. Since for high non-Newtonian parameter, the gradient of velocity profile differs considerably from that corresponding to low nonNewtonian numbers $(\Lambda \leq 0 \cdot 5)$, the gradient of the entropy generation number close to the pipe wall changes significantly. In this case, low viscous dissipation in this region is responsible for this behaviour.

Figure 5 shows total entropy generation number including heat transfer and fluid friction. It should be noted that the parameters $\left(\theta_{o}, \Gamma, \Lambda\right)$ are selected such that entropy generation numbers due to heat transfer and fluid friction becomes similar in magnitude. Consequently,

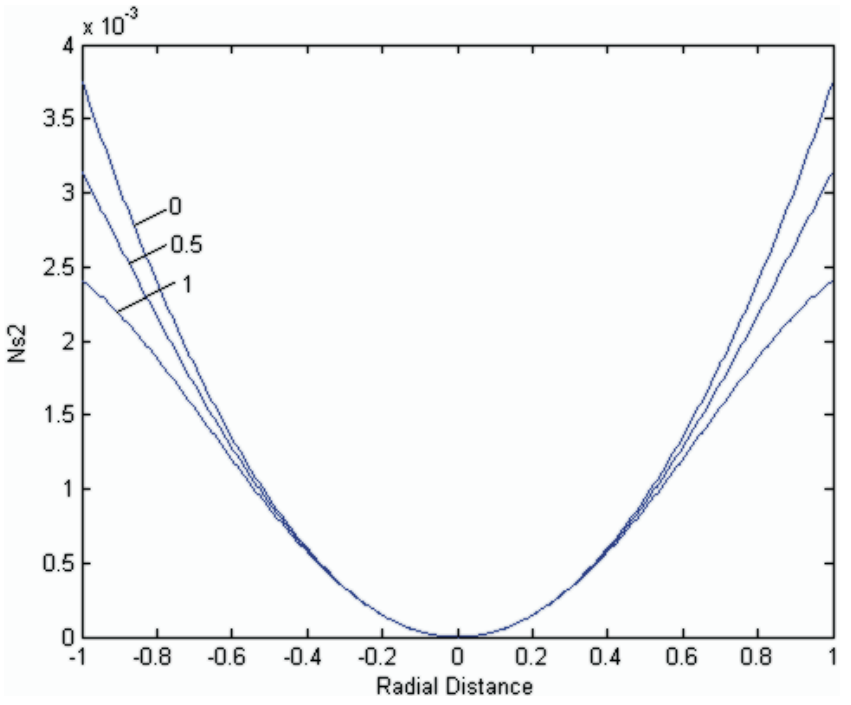

Figure 4. Entropy generation number due to fluid friction along the pipe diameter for different non-Newtonian parameters. $\Gamma=$ $1, C=-1$ and $\theta_{0}=0.015$. 


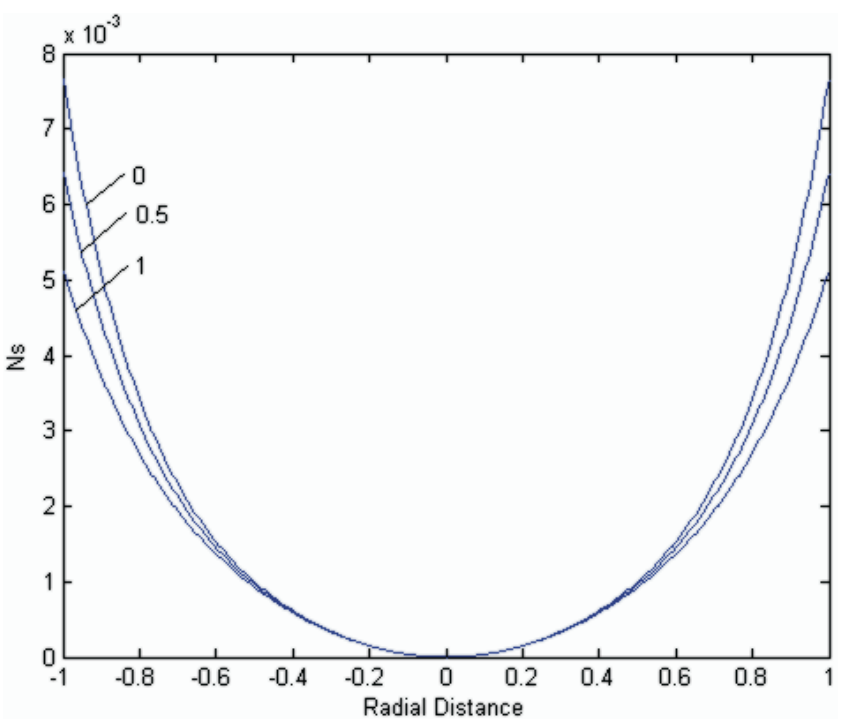

Figure 5. Entropy generation number due to heat transfer and fluid friction along the pipe diameter for different non-Newtonian parameters. $\Gamma=1, C=-1$ and $\theta_{0}=0 \cdot 015$.

curves in figure 5 represent both effects (heat transfer and fluid friction) on the total entropy generation number. Entropy generation number is high close to the pipe wall and reduces to zero at pipe centre. Increasing non-Newtonian parameter lowers the temperature and velocity gradients close to the pipe wall, which in turn results in reducing entropy generation number with increasing radial distance towards the pipe centre.

Figure 6 shows the influence of Brinkman number at different radial locations on the entropy generation number due to heat transfer and fluid friction. It should be noted that $r$ represents the dimensionless radius and $r=1$ is the pipe wall. Increasing Brinkman number enhances the fluid friction and heat transfer rates from pipe wall to the fluid (6). In this case, entropy generation number increases considerably with increasing Brinkman number. The influence

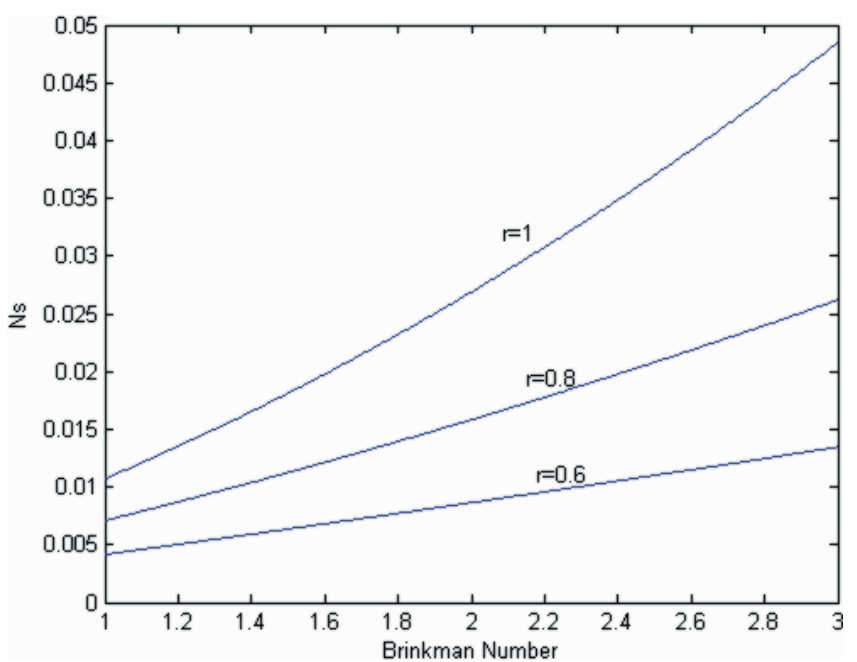

Figure 6. Entropy generation number due to heat transfer and fluid friction with Brinkman number at different radial locations in the pipe. $\Lambda=1, C=-1$ and $\theta_{0}=0.05$. 


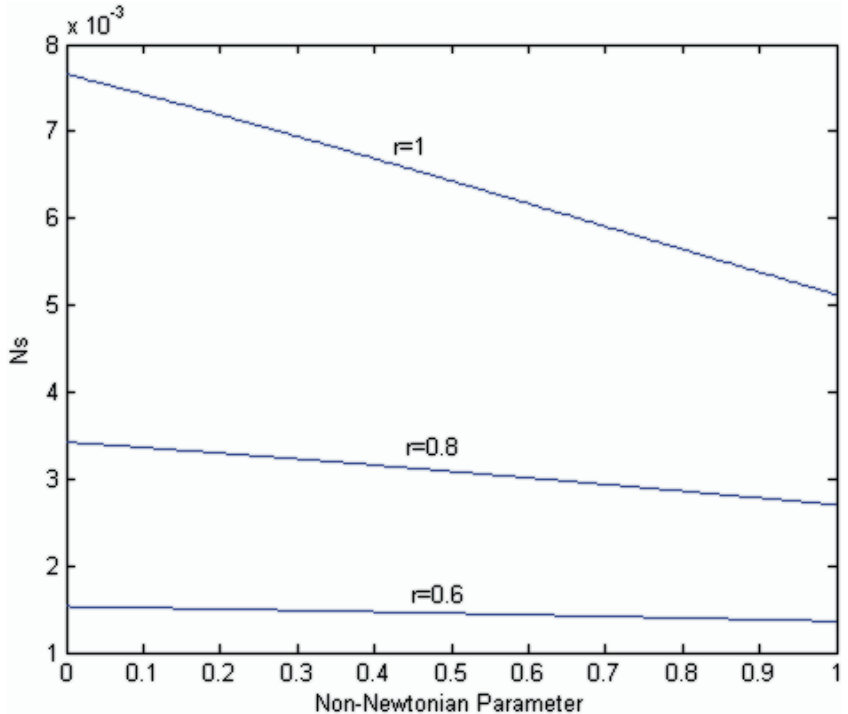

Figure 7. Entropy generation number due to heat transfer and fluid friction with non-Newtonian parameter at different radial locations in the pipe. $\Gamma=1, C=-1$ and $\theta_{0}=0.015$.

of Brinkman number on total entropy generation number is significant in that increasing Brinkman number enhances the entropy generation number.

Figure 7 shows total entropy generation number with non-Newtonian parameter at different radial locations in the pipe. In general, increasing non-Newtonian parameter lowers the entropy generation, which is more pronounced in the region close to the pipe wall. In this case, fluid friction reduces considerably in the pipe wall region. When the radial distance increases $20 \%$ away from the pipe wall, the influence of non-Newtonian parameter on the total entropy generation number becomes almost insignificant. In this case, entropy generation due to heat transfer reduces significantly, as well as the entropy generation due to fluid friction. Moreover, entropy generation due to heat transfer in the region between $20 \%$ of the pipe radius and the pipe centre becomes almost uniform at low values. Entropy generation due to fluid friction dominates the total entropy generation in this region. This situation is true for all non-Newtonian parameters. Consequently, total entropy generation number becomes almost steady with increasing non-Newtonian parameter for $r \leq 0 \cdot 8$.

\section{Conclusions}

Entropy generation due to non-Newtonian flow in a pipe is considered. The third grade fluid is accommodated in the analysis. The influence of non-Newtonian parameter and Brinkman number on entropy generation number is examined. It is found that increasing non-Newtonian parameter reduces the fluid friction in the region close to the pipe wall. This, in turn, lowers the entropy generation due to fluid friction in this region. The influence of non-Newtonian parameter on total entropy generation number is more pronounced in the surface region of the pipe wall where $r>0 \cdot 8$. However, entropy generation due to fluid friction dominates over entropy generation due to heat transfer for $r \leq 0 \cdot 8$. In this case, temperature profiles become almost uniform in this region. The influence of Brinkman number on the entropy generation number is significant for all radial locations in the pipe, except at the pipe centre. Moreover, increasing Brinkman number enhances the fluid friction and heat transfer rates; consequently, 
increasing Brinkman number increases entropy generation number. This situation is significant in the region close to the pipe wall.

\section{References}

Bejan A 1979 A study of entropy generation in fundamental convective heat transfer. ASME J Heat Transfer 101: 718-725

Bejan A 1994 Entropy generation through heat and fluid flow (New York: Wiley Interscience)

Bejan A 1995 Entropy generation minimization (New York: CRC Press)

Dunn J E, Fosdick R L 1974 Thermodynamics, stability, and boundedness of fluids of complexity 2 and fluids of second grade. Arch. Rat. Mech. Anal. 56: 191

Ekmann J M, Wildman D J, Chen J L S 1986 Laminar flow studies of highly loaded suspensions in horizontal pipes. Second Int. Symp, Slurry Flows, Anaheim, CA, ASME FED-38, 85

Fosdick R L, Rajagopal K R 1980 Thermodynamics and stability of fluids of third grade. Proc. $R$. Soc. Lond. A339: 351

Johnson G, Massoudi M, Rajagopal K R 1991 Flow of a fluid infused with solid particles through a pipe. Int. J. Engng. Sci. 29: 649

Mahmud S, Fraser R A 2002 Inherent irreversibility of channel and pipe flows for non-Newtonian fluids. Int. Commun. Heat Mass Transfer 29: 577-587

Massoudi M, Christie I 1995 Effect of variable viscosity and viscous dissipation on the flow of a third grade fluid in a pipe. Int. J. Non-Linear Mech. 30: 687-699

Pakdemirli M 1994 Conventional and multiple deck boundary layer approach to second and third grade fluids. Int. J. Engng. Sci. 32: 141-154

Peters, Baaijens 1997 Modelling of non-isothermal viscoelastic flows. J. Non-Newtonian Fluid Mech. 68: 205-224

Yilbas B S, Shuja S Z, Budair M O 1999 Second law analysis of a swirling flow in a circular duct with restriction. Int. J. Heat Mass Transfer 42: 4027-4041

Yurusoy M, Pakdemirli M 2002 Approximate analytical solutions for the flow of a third grade fluid in a pipe. Int. J. Non-Linear Mech. 37: 187-195 